(c) <2022>. This manuscript version is made available under the CC-BY-NC-ND 4.0 license http://creativecommons.org/licenses/by-nc-nd/4.0/

The definitive publisher version is available online at https://doi.org/ 10.1016/j.measurement.2021.110194 


\title{
Influence of particle morphology and concentration on the piezoresistivity of cement-based sensors with magneto-aligned nickel fillers
}

\begin{abstract}
:
Cement-based sensor with magneto-aligned nickel fillers is proven to have enhanced piezoresistivity compared with the sensor with randomized fillers. In this paper, the influence of particle morphology and concentration of nickel particles on the piezoresistive and mechanical properties of cement-based sensors, treated with and without magnetic field intervention, are investigated experimentally. Five categories of nickel particles with different average diameters are type N50 (50 nm), N500 (0.5 $\mu \mathrm{m})$, $\mathrm{F}(1 \mu \mathrm{m} \times 20 \mu \mathrm{m}$ flake), $\mathrm{T}(5 \mu \mathrm{m})$ and $\mathrm{U}(25 \mu \mathrm{m})$. The results indicate that the application of magnetic field enhances most of the piezoresistive performance and yields best piezoresistivity for the samples with type T nickel powder. Anisotropic piezoresistivity can be achieved under very low filler content (0.1 vol.\%) in N50 nano-scale nickel powder and cement composite, followed by the N500 and T nickel particles in 5 vol.\% content. Small particles with lower content have similar piezoresistive performance to the samples with large particles and higher concentration. Half of the samples achieve giant gauge factor (GF) over 500, two-thirds of which are aligned by magnetic field with anisotropic piezoresistive property. Samples with 5 vol.\% type T nickel content has the highest GF value, followed by the sample with 5 vol.\% type F nickel flakes and 10 vol.\% type $\mathrm{U}$ nickel powder. Mechanical strength drops with the increment of particle concentration.
\end{abstract}

Keywords: cement sensors; nickel; piezoresistivity; magnetic field; alignment.

\section{Introduction}

In recent years, cement based self-sensing composites/sensors have gained great research interest. By adding conductive filler materials into cement, mortar or concrete matrix, the composites have the 
ability of sensing external stress/strain and damage. Compared to conventional circuit chip sensors, these sensors are more easily integrated into the concrete matrix and thus have better compatibility with surrounding environment, also possess other advantages such as lower maintenance cost, higher durability, and larger sensing volume [1-3]. The merits offer great potentials in engineering applications such as structural health monitoring, weight in motion, traffic detection, and border security.

Currently, the state-of-the-art research work mainly focuses on three aspects: content and type of conductive fillers, effectiveness of dispersing filler materials in the matrix, the self-sensing behaviour under different external loadings [4]. The matrix of cement-based sensors is conventional building material such as cement paste, mortar or concrete. Due to the relative simplicity of matrix material, the focus of the studies then shifts to the filler materials which have great impact on the self-sensing behavior. The conductive or semiconductive fillers come from a wide variety materials including carbon fiber [5-7], carbon nanotubes [8,9], carbon nanofiber [10,11], carbon black [12, 13], graphite $[14,15]$ and graphene $[16,17]$. Besides theses carbon based materials, others such as conductive rubber [18], steel fibre [19], steel slag [20], nickel powder [21, 22], nano metal oxides [23], are incorporated to develop cement-based sensors as well. These fillers have great variation in morphology, thus can directly influence the piezoresistive properties and sensing performance. As shown in Fig.1, the fillers used in self-sensing can be categorised into three groups in aspect of shape including fibre, particle and flake, the size is from nano-scale to micro and macroscale. 


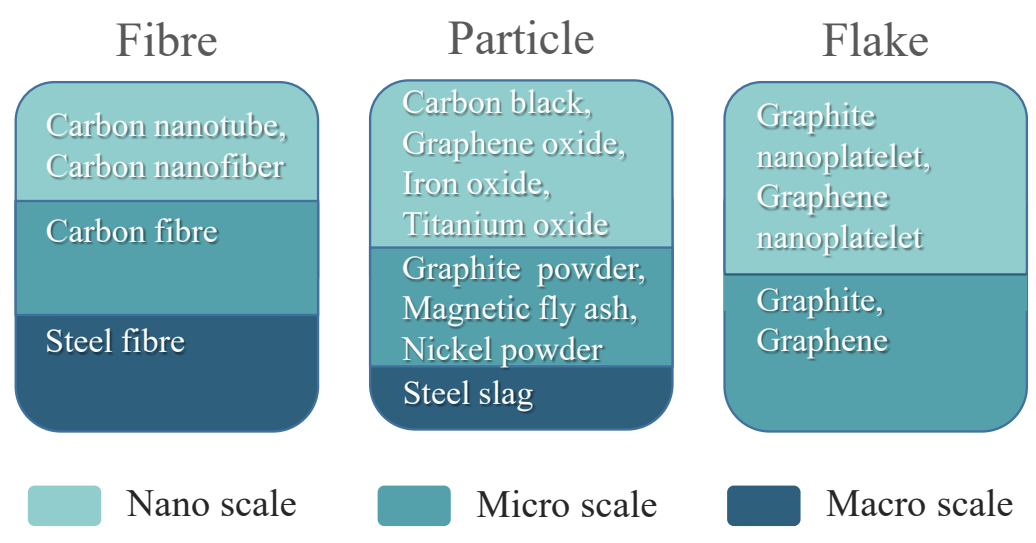

Fig. 1. Illustration of conductive filler materials used in relation to their scales and shapes.

The variant properties between filler materials have been explored in regards to their impact to the piezoresistivity and the preferences are mixed in terms of which one is a better option. Yoo et al [24] compared the piezoresistive behaviour among carbon nanotubes, graphite nanofibers and graphene oxide, and found that carbon nanotube was the most effective filler in promoting electrical conductivity when all the particles were used in the same proportion. While Al-Dahawi et al. [25] stated that carbon fiber seemed to be more beneficial than graphite nano-platelets and carbon nanotubes in terms of cost, dispersion process and electrical properties. In spite of the view point of Al-Dahawi et al., graphene nanoplatelets have gained more attention from many researchers recently [26, 27]. Firstly, compare to quasi-one-dimensional carbon nanotubes, graphene nanoplatelets have two-dimensional structure, and both sides of the platelets can contact to matrix material, which leads to a more sensitive function [28]. With certain thickness, graphene nanoplatelets have less chance of entanglement and agglomeration. The cost of graphene nanoplatelets is generally less than that of carbon nanotubes, which could be an essential factor for large scale industrial fabrication of self-sensing concrete [17]. Lastly, the required low level of concentration of graphene nanoplatelets (usually from $0.03 \%$ to $0.1 \%$ ) is another merit compared to carbon nanotubes [26-28]. 
Regarding nickel based conductive fillers, from nano-scale, submicron, micro to macro, they are in great morphology variety as well. Up to date, there has not been reported regarding using nano nickel powder and nickel flakes in developing self-sensing cement sensors. The nano nickel powder offers great potential to be used to stimulate piezoresistive performance in a low concentration due to its large specific surface area. The superior electrical conductivity and ferromagnetic properties of nickel flakes make it a great candidate to enhance self-sensing performance. Additionally, in literatures, the fillers are dispersed randomly into the matrix and formed 3D isotropic conductive network.

To enable useable piezoresistivity in cement-based sensors with isotropic conductive network, there are thresholds of the filler contents to meet which is usually above $10 \%$ or in many cases more than $20 \%$. However, such high filler contents leads to weak mechanical property which hinders the application of cement-based sensors in real applications. If only unidirectional sensing is required, anisotropic conductive network is desirable which could significantly reduce the required filler content. For example, anisotropic sensing property can be achieved by using magnetic field to align nickel particles into chain structure.

This study innovatively investigates the piezoresistivity of cement based composites with various nickel particles both with random and magneto-aligned patterns. It was found that most of the anisotropic cement sensors offers significant improvements in the piezoresistive performance compared to the random distributed filler sensors. By adjusting the parameters of magnetic field to an optimal level, for example, strength of $0.252 \mathrm{~T}$ and duration of $30 \mathrm{~s}$, same piezoresistivity could be achieved under lower particle concentration with comparable mechanical strength. Additionally, gaps in understanding the impact of particle morphology and concentration are bridged by investigating the piezoresistive, micro structure and mechanical properties of the cement composites with nanoscale nickel powder and nickel flakes. Firstly, the samples with various random and magneto-aligned nickel powder are fabricated, followed by the micro structure observation under scanning electron microscope (SEM). Then the piezoresistive performance is examined with cyclic and static 
compressive loading. Mechanical properties of the cement sensors are tested. The influences of particle morphology (including size) and concentration on piezoresistivity are discussed throughout the paper and comparison on piezoresistivity of different samples are summarized. Conclusions are drawn in the last part of the paper.

\section{Experimental}

\subsection{Raw material and sample fabrication}

In this research, general purpose cement is used as the matrix material of self-sensing cement-based composite. Five types of nickel powders are chosen as the conductive fillers: The first one is the spiky spherical shaped nickel powder with the diameter ranges from 3 to $7 \mu \mathrm{m}$ (model T123, Vale Canada Ltd.). In this research, it is defined as type $\mathrm{T}$ nickel powder. Other types of nickel powder with diameters from $50 \mathrm{~nm}, 0.5 \mu \mathrm{m}, 1 \mu \mathrm{m} \times 20 \mu \mathrm{m}$ flakes to $25 \mu \mathrm{m}$ are defined as N50, N500, type $\mathrm{F}$ and $\mathrm{U}$, respectively. Table 1 presents the specifications of the nickel powders. The morphology of the particles can be found in Fig. 2. Superplasticiser, a mixture of 1-10\% non-ionic surfactant (Amides, coco, $\mathrm{N}, \mathrm{N}-$ bis) and $<1 \%$ anionic surfactant (benzene sulfonic acid, dodecyl-, sodium salt)), helps to promote the flowability of the cement paste. Anti-foam solution (silicon emulsion with $<5 \%$ phosphates) is adopted to eliminate the air entraining effect caused by the superplasticiser during mixing.

Table 1. Specification of nickel powder.

\begin{tabular}{lllllll}
\hline $\begin{array}{l}\text { Nickel } \\
\text { powder } \\
\text { Model }\end{array}$ & $\begin{array}{l}\text { Average } \\
\text { diameter/ } \\
\text { thickness }\end{array}$ & Purity & $\begin{array}{l}\text { Specific } \\
\text { surface } \\
\text { area } \\
\left(\mathrm{m}^{2} / \mathrm{g}\right)\end{array}$ & $\begin{array}{l}\text { Bulk } \\
\text { density } \\
\left(\mathrm{g} / \mathrm{cm}^{3}\right)\end{array}$ & Shape & Colour \\
\hline N50 & $50 \mathrm{~nm}$ & $99.90 \%$ & 12 & 0.74 & Spherical & Black \\
N500 & $500 \mathrm{~nm}$ & $99.90 \%$ & 7.5 & 1.39 & Spherical & Black
\end{tabular}




\begin{tabular}{lllllll}
$\mathrm{F}$ & $1 \mu \mathrm{m} \times 20 \mu \mathrm{m}$ & $99.80 \%$ & 9.5 & 0.72 & Flakes & Silver Grey \\
$\mathrm{T}$ & $5 \mu \mathrm{m}$ & $99.80 \%$ & 0.4 & $1.8 \sim 2.7$ & Spiky spherical & Dark grey \\
$\mathrm{U}$ & $25 \mu \mathrm{m}$ & $99.50 \%$ & 0.025 & $3.2 \sim 5.5$ & Spherical & Grey \\
\hline
\end{tabular}

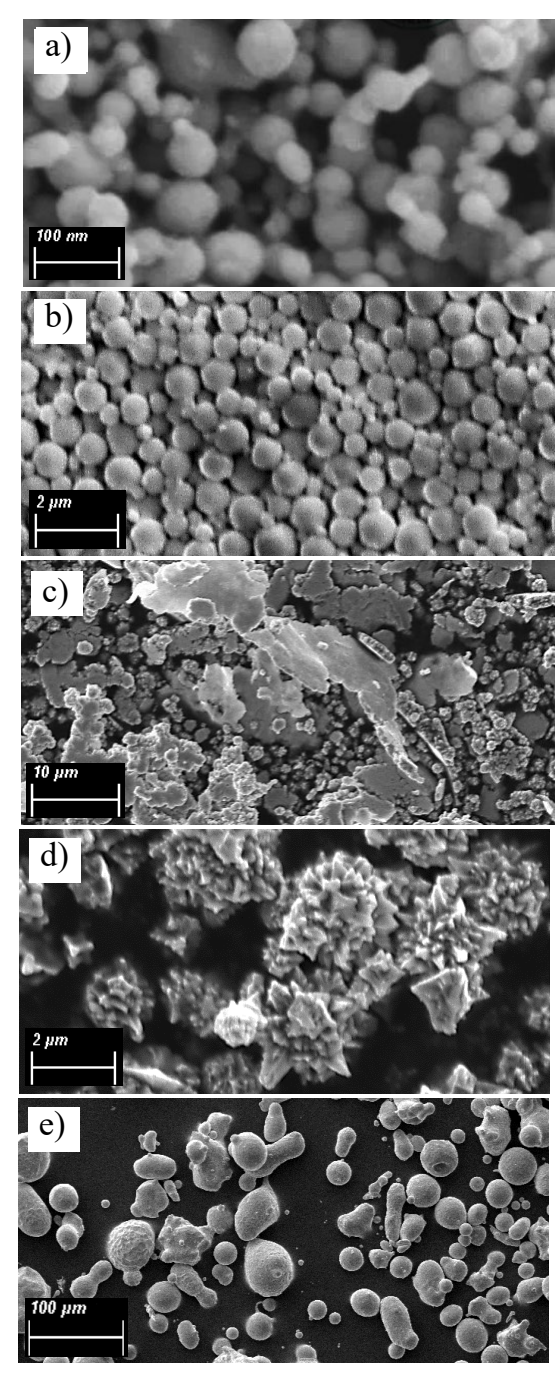

Fig. 2. The SEM images of the nickel particles used in this research.

$$
\text { a) N50, b) N500, c) P, d) T, e) U. }
$$

Cement composites with random nickel particles are fabricated as follows: in Fig. 3, superplasticiser and anti-foamer are added into water and stirred evenly. Then a process of 10-minute mechanical mixing and 30-minute sonication is applied after nickel powders are added into the solution to achieve a uniform dispersion. Next, cement is added into the solution and mixed with a Hobart mixer for 5 minutes. The paste is then poured into $2.5 \mathrm{~cm}$ customised moulds. Two electrodes (copper meshes in 
size of $1.5 \mathrm{~cm} \times 2.5 \mathrm{~cm}$ ) are vertically placed into the paste immediately. For the samples with chain patterns, the ferromagnetic particles are aligned by an electromagnet after the cement casting and this pattern is kept with the hydration process.

As shown in Table 2, the concentrations of nickel powders in the cement composites vary from $5 \%$ to $15 \%$ in volume fraction. Due to the high specific area of the nano scale particles of N50 nickel particles, its contents are reduced to $0.5 \%$ and $1.0 \%$ for effective dispersion. The composites with $15 \%$ nickel flakes have a very low mechanical strength and defined as invalid samples, then replaced by $7.5 \%$ concentration.

Table 2. Samples with different nickel powder contents.

\begin{tabular}{|c|c|c|c|c|c|c|}
\hline & & Sample Name & & & $\begin{array}{l}\text { Nickel powder } \\
\text { concentration } \\
(\text { vol. } \%)\end{array}$ & $\begin{array}{l}\text { Sample } \\
\text { quantity }\end{array}$ \\
\hline N50-R5 (0.5\%) & N500-R5 & F-R5 & T-R5 & U-R5 & 5.0 & 9 \\
\hline N50-A5 $(0.5 \%)$ & N500-A5 & F-A5 & T-A5 & U-A5 & 5.0 & 9 \\
\hline N50-R10 (1\%) & N500-R10 & F-R7 (7.5\%) & T-R10 & U-R10 & 10.0 & 9 \\
\hline N50-A10 (1\%) & N500-A10 & F-A7 (7.5\%) & $\mathrm{T}-\mathrm{A} 10$ & U-A10 & 10.0 & 9 \\
\hline - & N500-R15 & F-R10 & T-R15 & U-R15 & 15.0 & 9 \\
\hline- & N500-A15 & F-A10 & $\mathrm{T}-\mathrm{A} 15$ & U-A15 & 15.0 & 9 \\
\hline
\end{tabular}

Note: R-fillers are dispersed randomly; A-fillers are aligned to chains under magnet field.

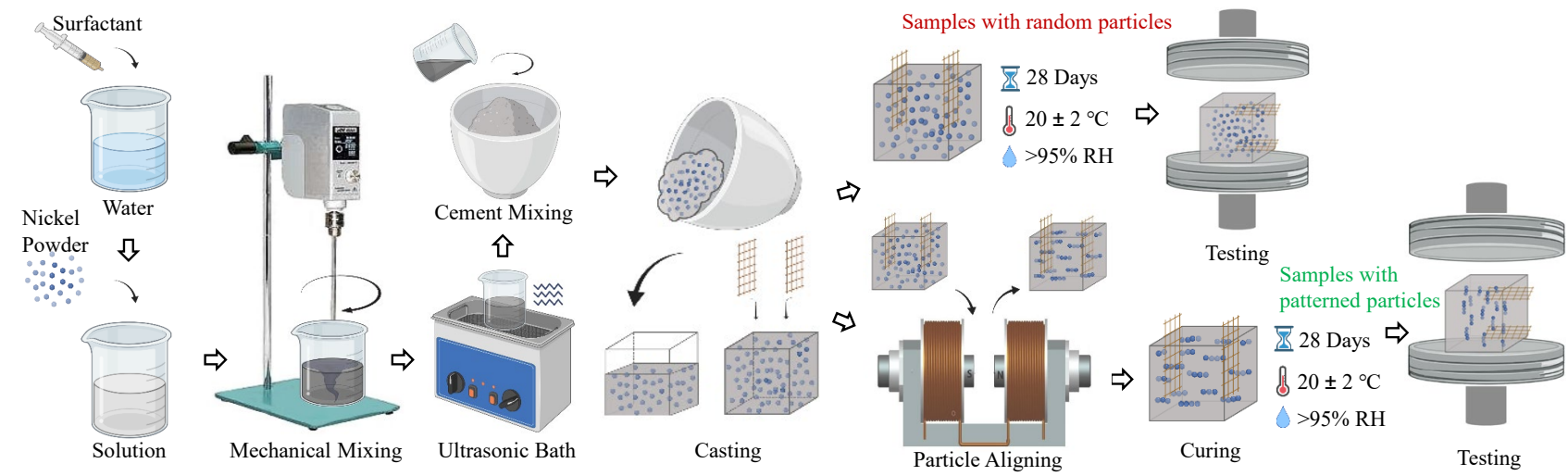

Fig. 3. Flow chart of fabricating cement-based composites with magneto-aligned nickel powders. 
To ensure the consistent in flowability and viscosity for cementitious material with different fillers, the workability is measured and compared using a flow table test according to ASTM C230/C230M21. By adjusting the water to cement ratio and superplasticiser concentration, the composites flow diameters are controlled in similar values of the samples with various particles and content levels.

\subsection{Magnetic field parameter selection}

The key factors affecting the formation of the chain structure in the cement based sensors are magnetic field strength and its applied duration. Several tests are carried out to find an ideal magnetic field strength. When the strength is insufficient, e.g., under $0.05 \mathrm{~T}$, the chian pattern was not formed or partially formed. A magnetic field beyond $1.0 \mathrm{~T}$ is not desirable since samples will bulge due to the magnetostrictive effect of the nickel particles. When a magnetic field between $0.5 \mathrm{~T}$ to $1.0 \mathrm{~T}$ is applied, the particles in the formed chain structure tend to adjacent or connect to each other. The direct contact dominated network leads to a reduced effect on piezoresistive performance. Thus, the magnetic field strength is controlled from $0.085 \mathrm{~T}$ to $0.252 \mathrm{~T}$, which is achieved through adjustment of the input current on the electromagnet. In terms of the effect of magnetic field duration, it has less influential compared to that of magnetic field strength. Indication found here is that longer magnetic field duration appears to be unnecessary for this type of cement-based sensor. Taking the samples with $5 \%$ type $\mathrm{T}$ nickel particles as an example, the chain formations formed by various magnetic field strength and duration are illustrated in Fig. 4. It can be seen that with the increasing of strength, from $0.085 \mathrm{~T}, 0.167$ $\mathrm{T}$ to $0.252 \mathrm{~T}$, the chains gradually forms and the length of chains grows longer. In the second row (varying application duration), the short chains have tendency of shrinking under $90 \mathrm{~s}$ duration. Therefore, $0.252 \mathrm{~T}$ and $30 \mathrm{~s}$ are chosen as an optimal combination to align the nickel particles in the following test. 


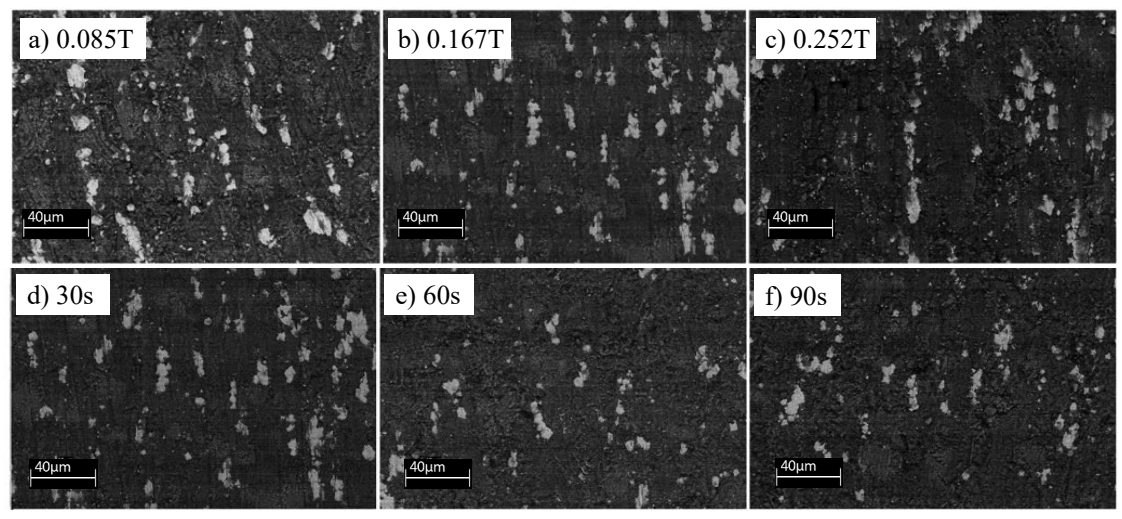

Fig. 4. SEM image of the chain structure in samples with 5 vol.\% type T nickel particles formed by different magnitudes of magnetic intensity and duration (from $0.085 \mathrm{~T}$ to $0.252 \mathrm{~T}, 30 \mathrm{~s}$ to $90 \mathrm{~s}$ duration).

\subsection{Experiment set-up}

The set-up of piezoresistive test can be found in Fig. 5. Static and cyclic compressive loadings with peak values of $24 \mathrm{MPa}$ and $10 \mathrm{MPa}$ respectively, are applied by Shimadzu AG-X50 universal testing machine. The change of electrical resistance is recorded through a multimeter (Siglent SDM3055 51/2 Digit Dual-Display Digital Multimeter) synchronously while loading. Mechanical strength is tested under Shimadzu UH-500 universal testing machine. All the tests are under the environmental condition of $22{ }^{\circ} \mathrm{C}$ and $50 \%$ relative humidity. Three cycles of pre-loading with $12 \mathrm{MPa}$ peak value within elastic range is to eliminate the brittle fractures and stabilise the contact resistance. Two strain gauges are attached to both sides of the cubic samples for balancing results. 


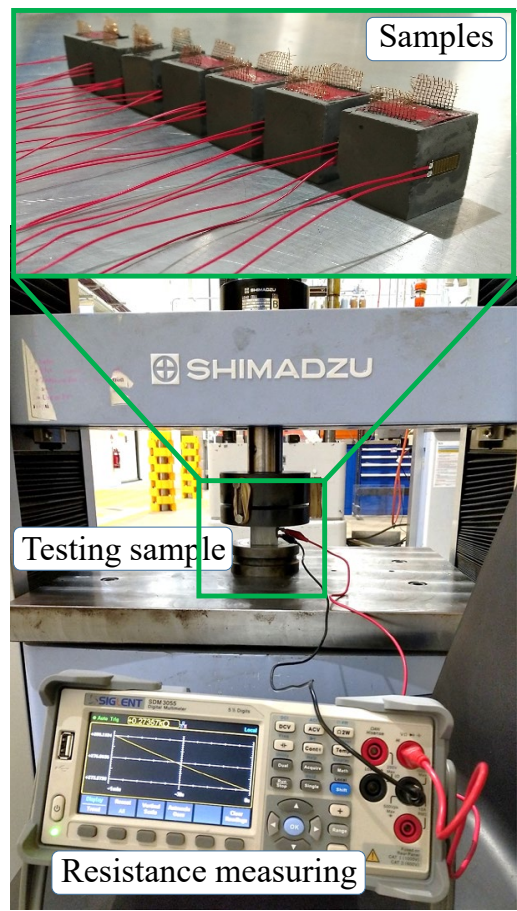

Fig. 5. Set-up of piezoresistive properties measurement.

\section{Result and discussion}

\subsection{Morphology and particle distribution}

To understand the aligned particles microcosmic distributions, and their influence on piezoresistive and mechanical properties, samples are observed under SEM. The type N500, T and U nickel powders form clear patterns under the magnetic field of $0.252 \mathrm{~T}$ magnitude. With the increasing of particle concentration, the length of chains is getting longer. As shown in Fig. 6, the length of the three samples are $7.6 \mu \mathrm{m}, 102.4 \mu \mathrm{m}$ and $109.0 \mu \mathrm{m}$, respectively at $5 \%$ volume fraction. These values increase to 13.5 $\mu \mathrm{m}, 105.1 \mu \mathrm{m}$ and $140.0 \mu \mathrm{m}$ at $10 \mathrm{vol} . \%$ content. With further rising to $15 \mathrm{vol} . \%$, the microscope image is filled with micro particles and the chains are indistinquishable due to the influence of scatters particles and increased total particle number. Only type U nickel particles exhibit a clear chains structure with an average chain length of $169.0 \mu \mathrm{m}$ due to its bigger diameter at this content. For the 
samples with N50 nano scale nickel particles, although the concentration is not high compared to other type samples, small aggregations still can be found in the composites which result from the high specific surface area and Van der Waals force between the particulates. Moreover, if no clear chain patterns are found under the SEM image, it suggests an ineffective particle distribution and less sufficient conductive network. In terms of the samples with type F nickel flakes, they present a well dispersed mixture from 5 vol. $\%$ to 15 vol.\% contents. No particle agglomerations are found in the scanning samples. The conductive network is effective from the aspect of filler dispersion which means the potential of high performance in piezoresistivity.

In general, the composites with N500, T123 and U25 nickel powders exhibit clear chain patterns under the SEM images. No chains are found in the N50 samples due to the aggregate effect and small particle size. The F type nickel flake samples have the piezoresistive advantage of great deformation and superior electrical conductivity, however the mechanical strength may be compromised owing to the special plate shape. All the samples with 15 vol.\% particle concentration do not obtain a great visualising performance in the form of chains.

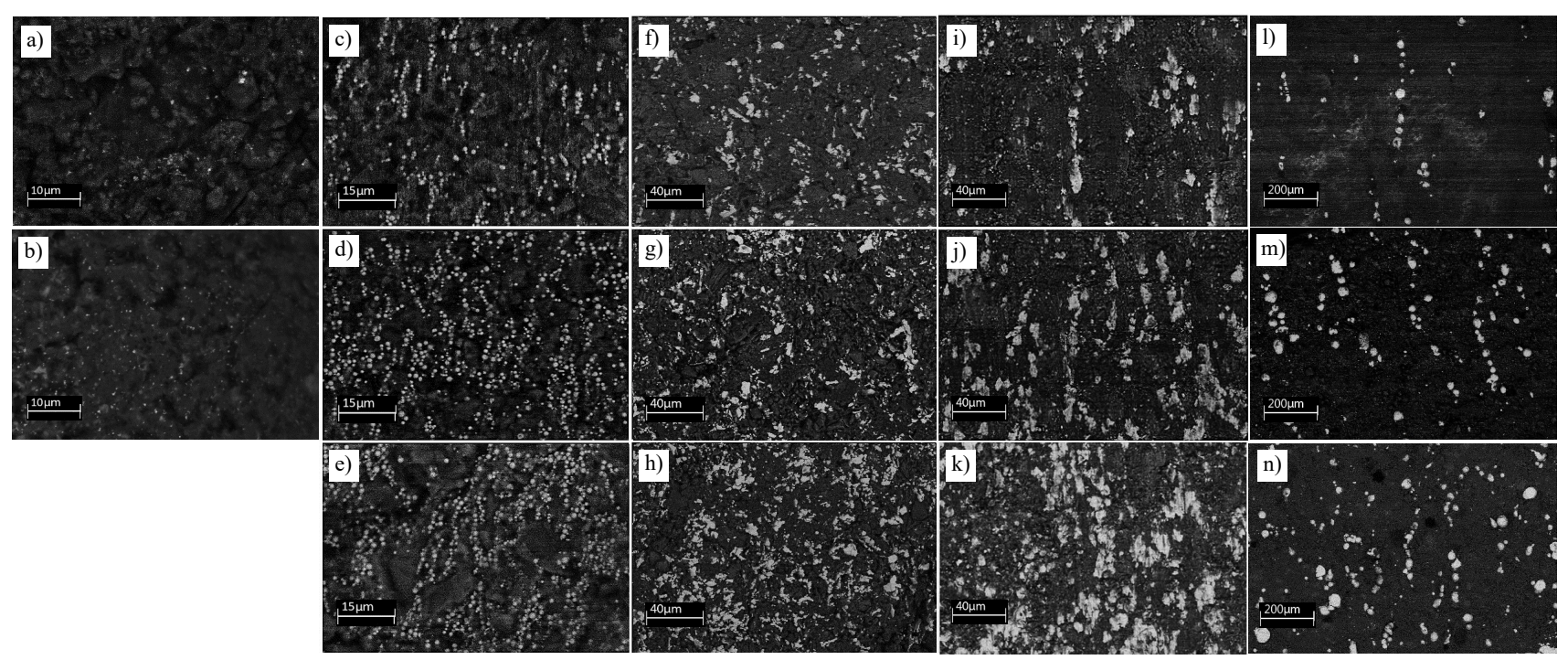


Fig. 6. SEM images of chain structures formed by different nickel powder in 5\%,10\% and 15\% volume fraction content. a) N50-5 (0.5\%), b) N50-10 (1.0\%), c) N500-5, d) N500-10, e) N500-15, f)

$$
\text { F-5, g) F-7, h) F-10, i) T-5, j) T-10, k) T-15, 1) U-5, m) U-10, n) U-15. }
$$

\subsection{Piezoresistive performance}

\subsubsection{Isotropic and anisotropic piezoresistive property of sensors under cyclic compressive loading}

Cyclic testing in the elastic region is used to examine the piezoresistivity of the sensors repeatability under repetitive loading and the ability to recover while unloading. To compare the piezoresistive performance of the cement composites with different nickel particles and the anisotropic piezoresistivity under the unidirectional network formed by these particle chains, three-cyclic compressive loading within elastic range and one high stress static compressive loading are introduced.

As shown in Fig. 7-12, fractional change in resistivity (FCR) of all samples follows the compressive loading synchronously. It decreases with the loading process, and gradually drops in the unloading. In Fig. $7 \mathrm{a}$ and $7 \mathrm{~b}$, the samples with 0.5 vol.\% and 1.0 vol.\% N50 randomized nano-scale nickel powder have FCR values of $-6.9 \%$ and $-12.5 \%$, respectively. After the conductive fillers are aligned by the magnetic field, the FCR is doubled to $-12.3 \%$ and $-23.0 \%$. It is worth noting that similar FCRs are achieved in these samples with extreme low particles concentration, which are only one-tenth of the samples with micro size particles. The specific surface area of N50 reaches $12 \mathrm{~m}^{2} / \mathrm{g}$, which is 480 times of $U$ type nickel powder. From economical consideration, particles with smaller diameter is the first choice. However, it brings side-effect on dispersion. Due to the high specific surface area and attractive force between the particles, high powder and long-time sonication process are necessary to disperse these nano particles into the solution before cement mixing. In these research, although the process is carried out carefully, small agglomeration that can reduce the piezoresistive performance 
and mechanical strength still inevitable exist in the composites. Moreover, after the particle alignment, the improvement of FCR is insignificant, only two orders of the samples compared with random fillers.
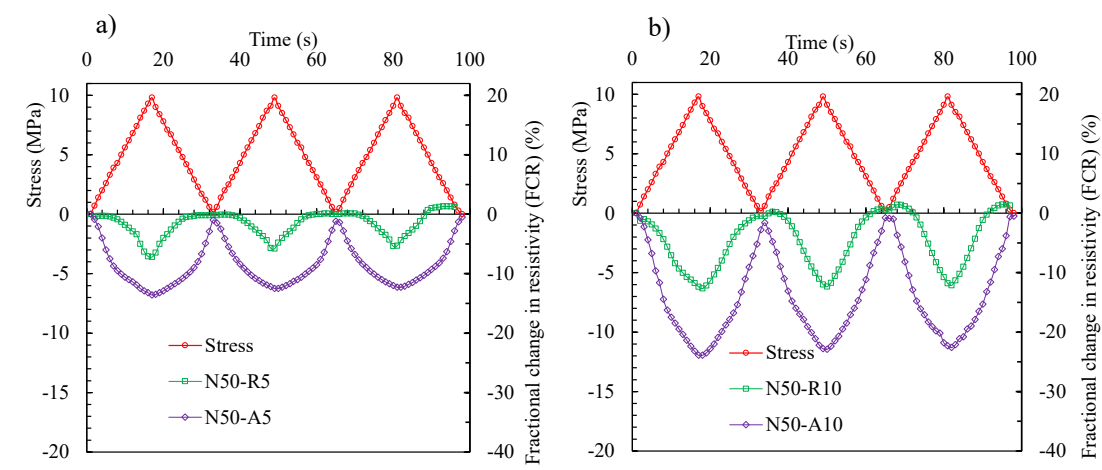

Fig. 7. Fractional change of resistivity of cement-based composites with 0.5 vol.\% and 1.0 vol.\% N50 nickel powder in two different conductive networks (R-sample with random dispersed fillers, Asample with chain pattern particles in the direction of magnetic field).

When the nickel particle diameter increases 10 times to $0.5 \mu \mathrm{m}$, the piezoresistive property of the composites with N500 nickel powder is quite different from those with N50 particles. As shown in Fig. 8 , the FCR of samples with random fillers keeps decreasing, from $-5.8 \%$ to $-6.5 \%$ and reaches $-33.8 \%$ when the particle concentration rises from 5 vol.\%, 10 vol.\% to 15 vol.\%. This decreasing tendency indicates the enhancement of the conductive network and the great drop shows the content is approaching the percolation threshold which is the most optimal and effective filler content for piezoresistive performance. On the contrary, the FCR of the samples with aligned nickel particles shows an opposite trend. It gradually increases from $-15.0 \%,-11.2 \%$ to $-7.5 \%$ beyond a volume concentration of $15 \mathrm{vol} \%$. The rising indicates that the conductive paths are getting saturated with further increasing of the particle numbers. With the alignment facilitated by magnetic field, the percolation threshold may be shift toward to $5 \%$ vol. $\%$ or under it. 

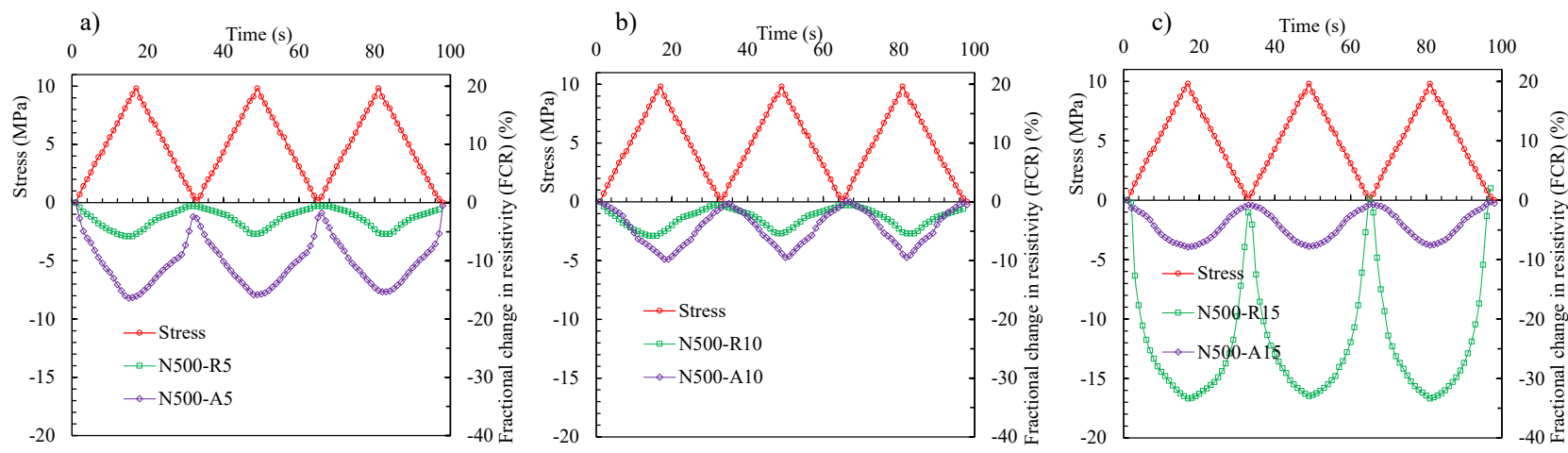

Fig. 8. Fractional change of resistivity of cement-based composites with 5 vol.\%, 10 vol.\% and 15 vol.\% N500 nickel powder in two different conductive networks (R-sample with random dispersed fillers, A-sample with chain pattern particles in the direction of magnetic field).

Regarding the samples with F type nickel flakes in Fig. 9, the FCR of samples with 5 vol.\% particles is improved from $-7.5 \%$ to $-20.1 \%$ after the intervention of magnetic field. When the filler content rises to 10 vol. $\%$ and 15 vol. $\%$, the values are $-30.5 \%,-29.8 \%,-31.9 \%$ and $-32 \%$, respectively. There is nearly no distinct variation between the samples with random and aligned particles. From the SEM images, it can be concluded that the conductive network formed by nickel flakes under magnetic field is very similar to a random distributed system rather than aligned into chians. These flakes are under random distribution in the initial stage. Under magnetic field, the magnetization of magnetic dipoles makes the flakes rotate until the balance is reached, as shown in Fig. 10a. This rotating motion $\mathrm{T}$ is random due to the magnetic dipoles moment and irregular in-plane shape, and affected by the intensity of external magnetic field and viscosity of the cement matrix. Because the flakes have a large in-plane size, whether aligned or not, they could bridge the chains and reduce the anisotropic piezoresistive performance (Fig. 10b). Notably, this type cement sensor can achieve a high FCR of $-31 \%$ under a relative low concentration $(7.5$ vol.\%). 

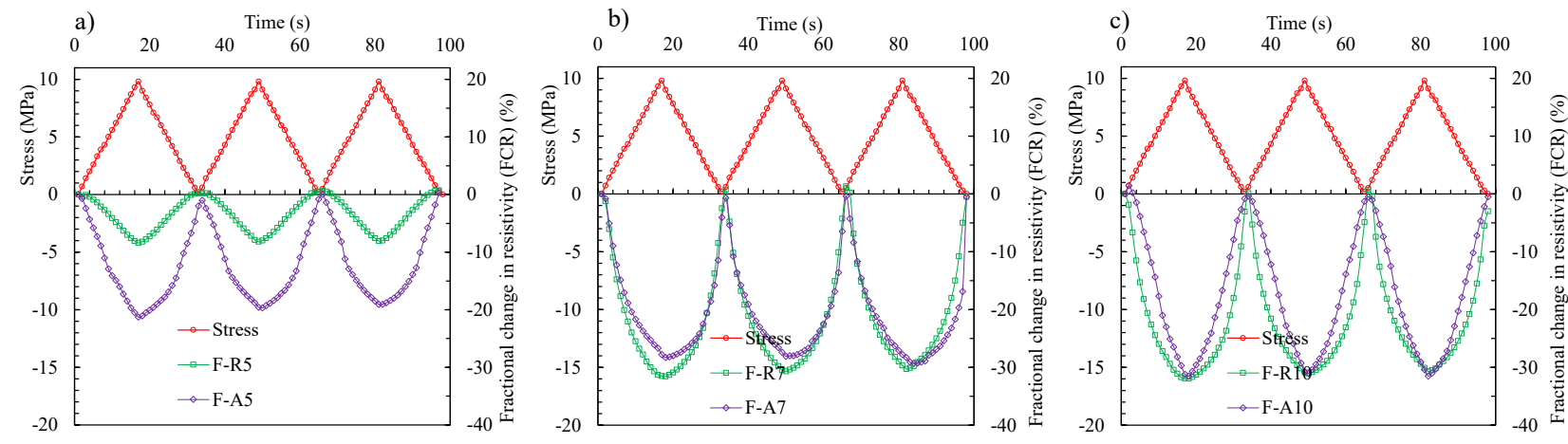

Fig. 9. Fractional change of resistivity of cement-based composites with 5 vol. $\%, 7.5$ vol. $\%$ and 10 vol.\% type F nickel flakes in two different conductive networks (R-sample with random dispersed fillers, A-sample with chain pattern particles in the direction of magnetic field).
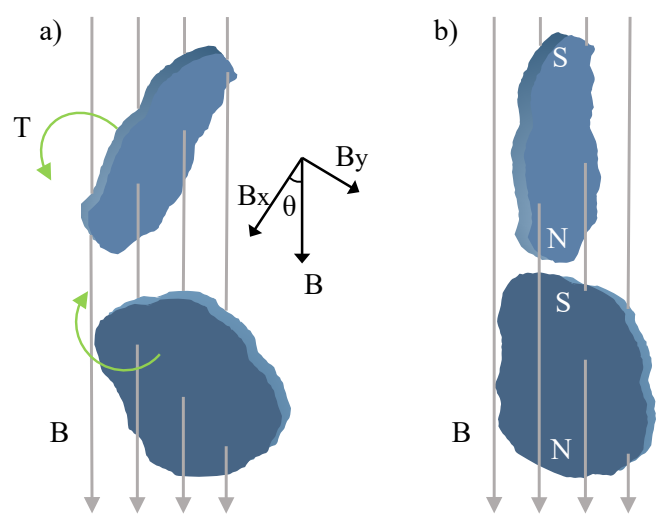

Fig. 10. The alignment of nickel flakes under magnetic field.

Fig. 11 illustrates the pirezoresistive performance of the samples with 5 vol. $\%, 10$ vol. $\%$ and 15 vol. $\%$ T123 nickel powder, respectively. The trend is similar to that of the samples with N500 nickel particles. The FCR values of samples with random dispersed particles decreases from $-5.2 \%,-13.3 \%$ to $-14.2 \%$ and reaches a peak value at $-32.1 \%$, then slowly rises to $-30.1 \%$ and $-25.2 \%$ for the particles rearranged by the magnetic field. The distinction of the FCR between random and aligned particles is significant. Especially in the low concentration, e.g. 5 vol. \%, it increases 6.2 times after magnetic field intervention. This high performance may lie in the unique morphology of the T123 nickel powder. 
Different from other spherical nickel powder, it has a rough surface texture and thorn-like points. Since the electrical field has a scale relationship with the needle-like shape size [29], this can enhance the field emission effect which explaines by electrons quantum mechanical tunnelling up to 1000 times [30]. It is much greater than on the smooth surface of other spherical particles. Field emission effect is generated by the enhanced local electrical field around the nanotips and makes the electrons tunnel through the barrier much easier. Under compressive loading, the electrons hopping effect takes place along the unidirectional conductive network altered by magnetic field, resulting an effective participation of conductive fillers and the decrease of percolation threshold [31]. Therefore, electrical conductivity of type T nickel and cement composites is strengthened by the field emission effect, and currently the piezoresistivity is the most significant in these five types of composites.
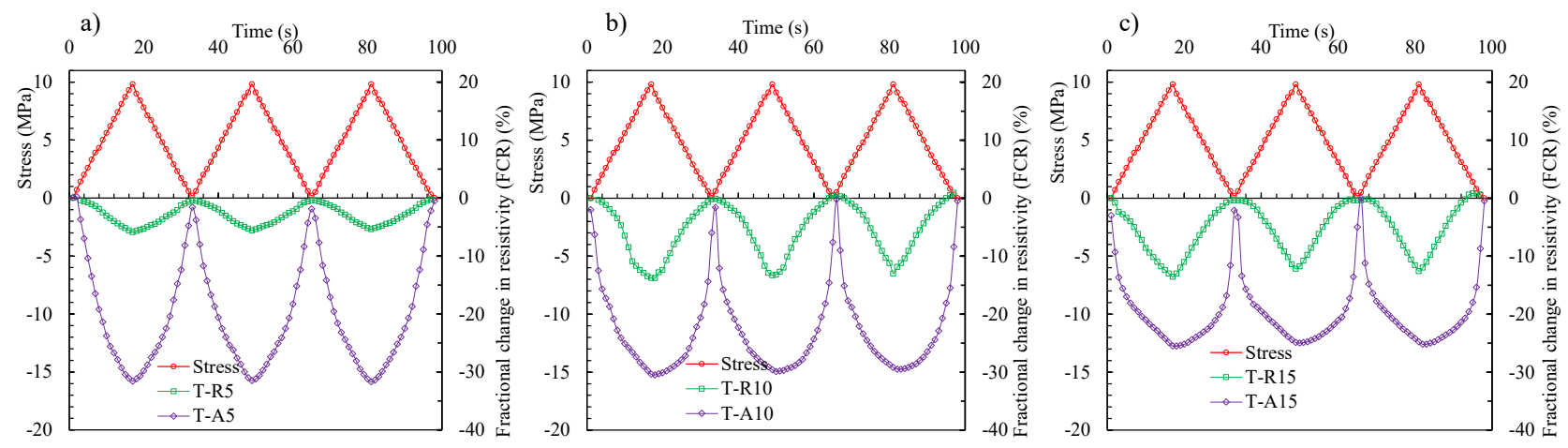

Fig. 11. Fractional change of resistivity of cement-based composites with 5 vol.\%, 10 vol.\% and 15 vol.\% type T nickel powder in two different conductive networks (R-sample with random dispersed fillers, A-sample with chain pattern particles in the direction of magnetic field).

The type U nickel particles have the largest diameter $(25 \mu \mathrm{m}), 500$ time of the nano-scale nickel powder. From the FCR charts in Fig. 12, the samples with 10 vol. \% and 15 vol.\% random and patterned particles have similar FCR values (approximately -21\%), whose distinction between the two pattern fillers are not as significant as other four types nickel powders. The aligned samples' FCR is even smaller than that of the random distributed particles under 5 vol.\% content. It can be concluded that when the particles size increases to $25 \mu \mathrm{m}$, anisotropic effect from the magnetic field is gradually 
disappeared. Especially under lower filler content, the quantity of the particles per unit volume decreases. Although the particles are aligned, the distance between each particulates is increased and the chians cannot connect to each other, in other words, the conductive network is formed insufficiently either for electron hopping or direct contact, leading to the poor piezoresistive performance. Even though the volume fraction rising to $10 \%$ and $15 \%$, the total chains number is reduced owing to the unit particle volume mentioned above.
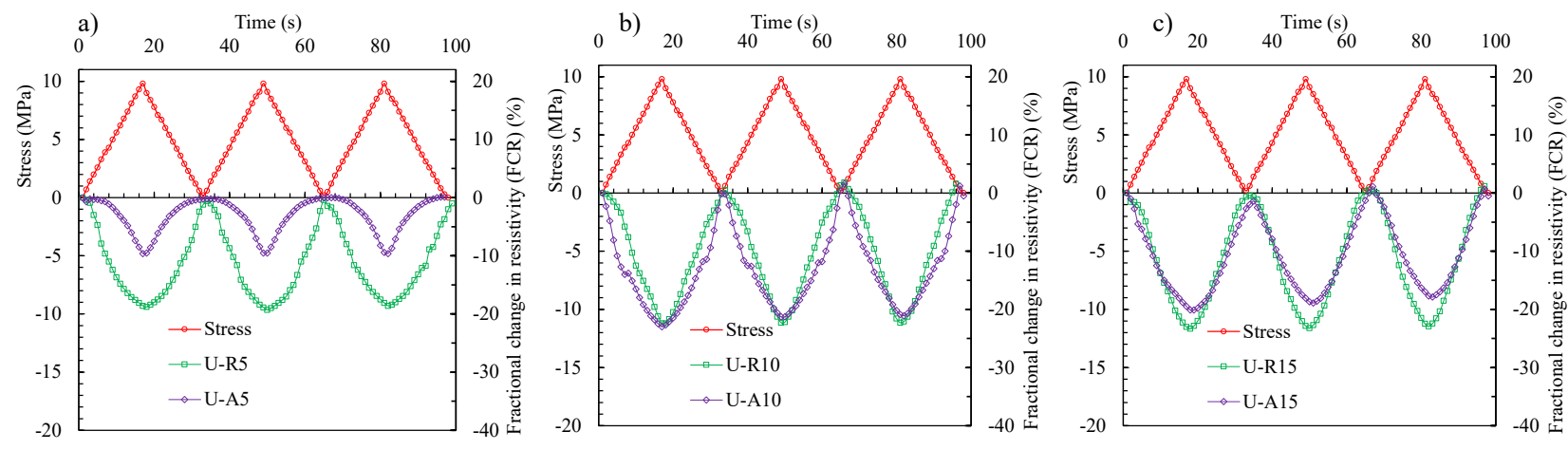

Fig. 12. Fractional change of resistivity of cement-based composites with 5 vol.\%, 10 vol.\% and 15 vol.\% Type U nickel powder in two different conductive networks (R-sample with random dispersed fillers, A-sample with chain pattern particles in the direction of magnetic field).

\subsubsection{Sensors under high static compressive loading}

Here, a static compressive loading with higher stress up to $24 \mathrm{MPa}$ is carried out to evaluate the piezoresistive performande of the sensors under high static load. From Fig. 13, it can be seen that most of the FCR curves drop rapidly in the beginning and then gradually slow down, exhibiting a non-linear pattern. The non-linearity is more obvious in high filler concentration. This may have been resulted from the differences in the conductive network formed by the nickel particles. With increasing compressive loading, more contact points between particles are established, inter-particle distance shrinks and the resistance drops rapidly. After the network reaches its threshold, i.e., with a certain 
filler concentration, further increasing contact points have less contribution to the conductive function, leading to the slowdown of the curves. The small fluctuations on the curves, especially in the high stress stage, for example, the convex and concave points denote the sudden increment and drop of electrical resistance, respectively. Such change in the conductive network resulted in the improvement in the accuracy of the sensors. The defects can be detected instantly, for instance, under loading, voids' sudden compression can lead to the particle interface reduction, from a far and insulated state to a close distance for electrons hopping, as shown in Fig. 14a and 14b. On the FCR curve, it is displayed as concave fluctuation. On the other hand, the convex fluctuation presents sudden cracks that break the conductive network and lift the resistance (Fig. 13c).
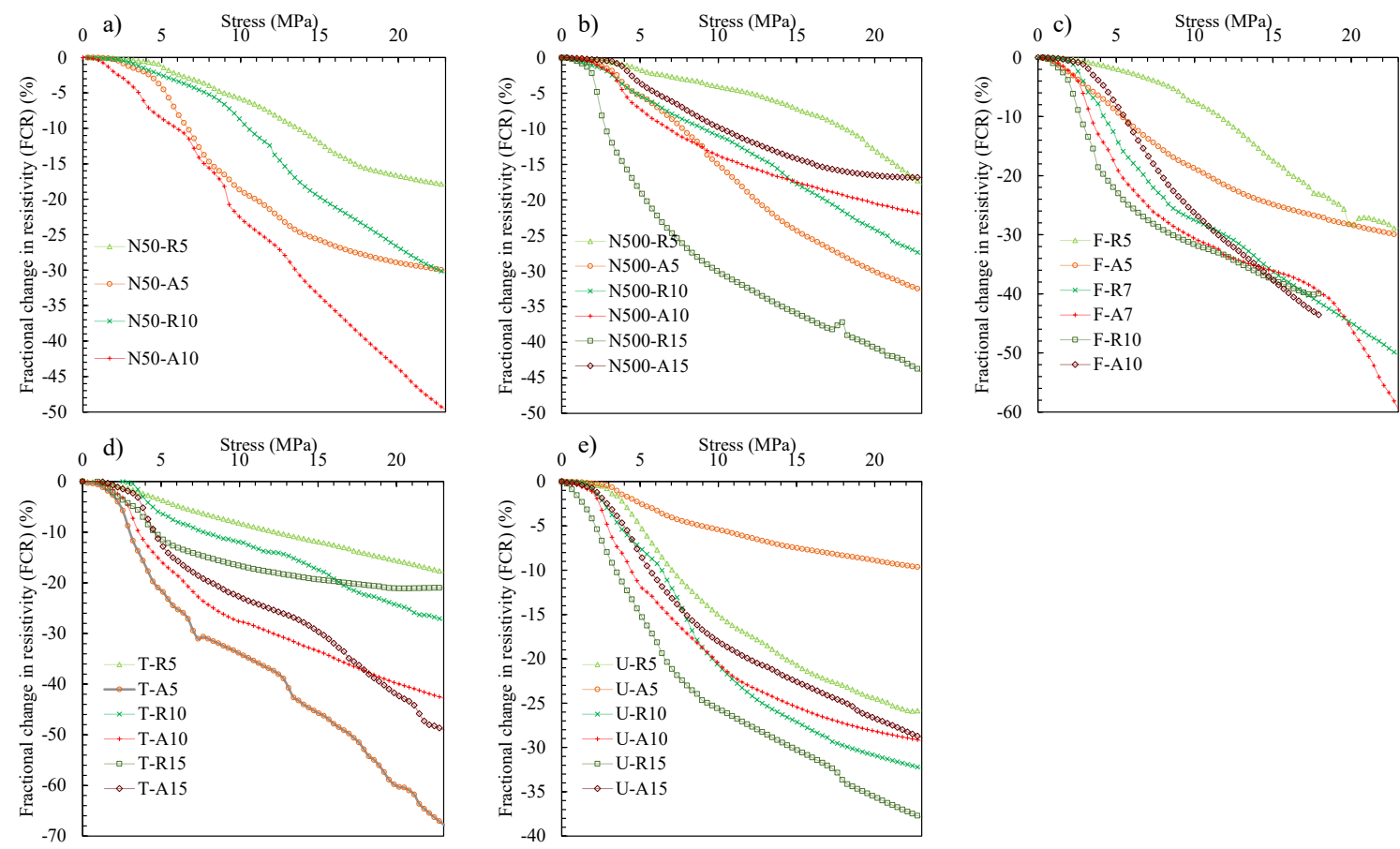

Fig. 13. Piezoresistivive performance of cement-based nickel powder composites under high stress static loading. (R-sample with random dispersed fillers, A-sample with chain pattern particles in the direction of magnetic field). a) N50, b) N500, c) type F, d) type T, e) type U. 

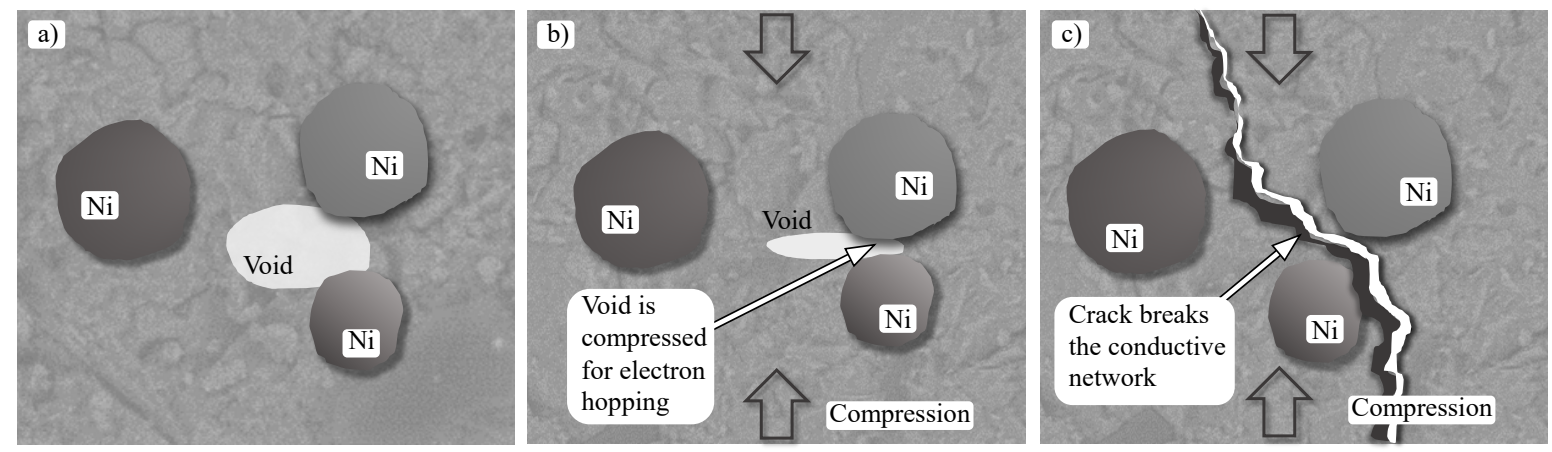

Fig. 14. Illustration of nickel powder under compressive loading and the change of conductive network.

\subsubsection{Mechanical properties}

\subsubsection{Compressive strength}

The compressive strength is an important factor of cement based composites. When these sensors are embedded into structures, high strength ensures good compatibility with the hosting structure in sustaining large loadings. While sensors with relatively low strength can be used in a low stress area within elastic deformation.

Strength test results show that the compressive strength of the sample decreases with the rising of nickel filler concentration for all types nickel powder (Fig. 15). The original 28-day ultimate strength of reference plain cement paste is $62.3 \mathrm{MPa}$. The sample with 10 vol.\% nickel flakes has the lowest strength of $32.5 \mathrm{MPa}$. All samples with 15 vol.\% nickel content are associated with weak strength. However, in lower concentration, e.g., 5 vol.\%, the strength is acceptable except the sample with nickel flakes. With a significant sensitivity and piezoresistive property, the $\mathrm{T}$ type nickel composites have excellent performance. Generally, the merit of magnetic field induced samples is that it can achieved same piezoressitive performance under a much lower nickel particle concentration, e.g., 5 vol.\%, rather than a much higher filler content, i.e., 15 vol. \%, for the samples with random fillers. It 
means the compressive strength is guaranteed, or the samples can gain high sensing property and mechanical strength simultaneously.
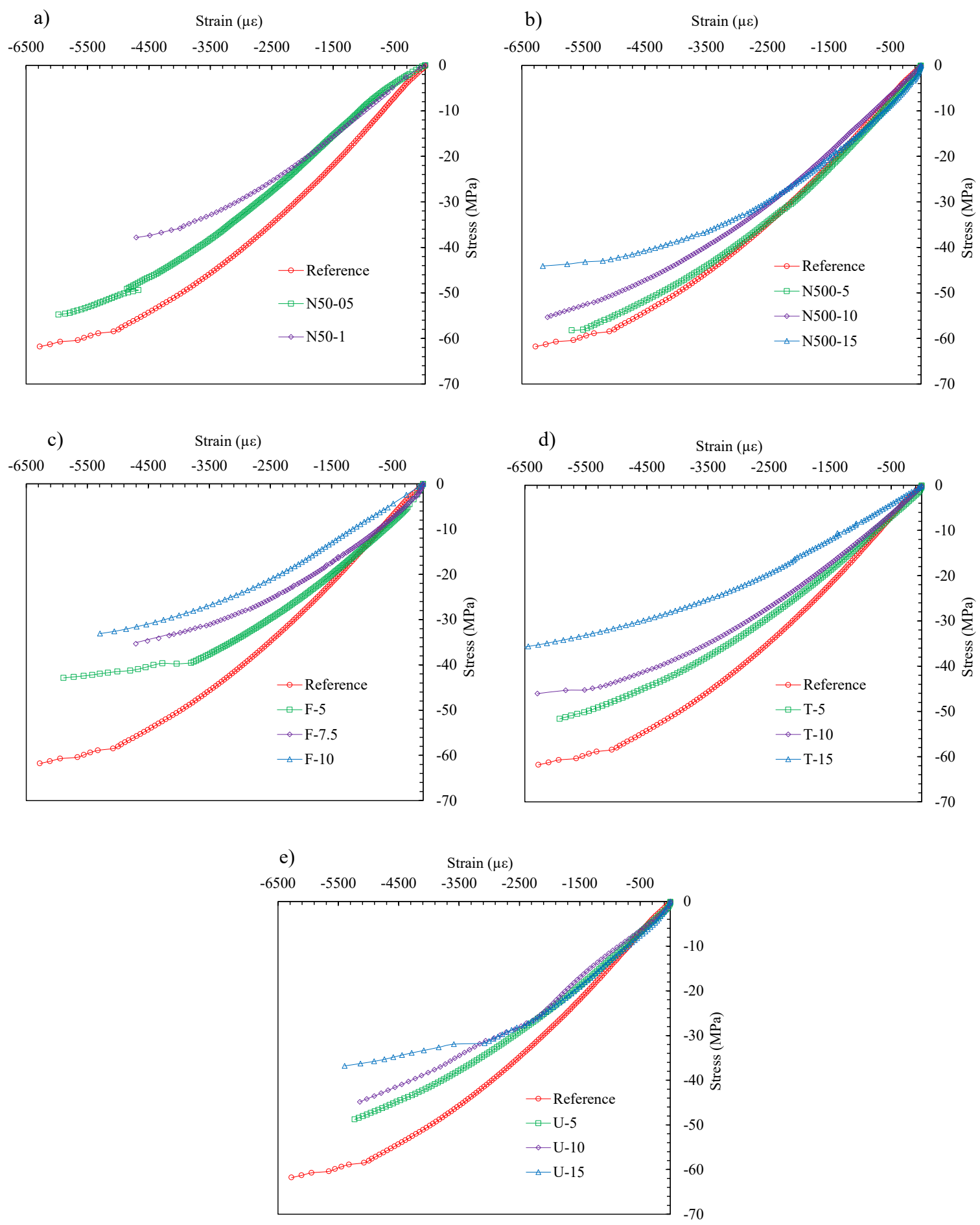

Fig. 15. Compressive strength of cement-based composites with different nickel powder in 5\%, 10\% and $15 \%$ particle content. a) N50, b) N500, c) type P, d) type T, e) type U. (The type F contents are

$$
5 \%, 7.5 \% \text { and } 10 \%)
$$


There are challenges found to be addressed in this research. For example, owing to the greater density of nickel and specific surface area, the N50 nickel particle is not easy to disperse into solution, agglomerations can be found in the composites (Fig. 16a). This will become more serious with the rising of particle concentration. Therefore, higher concentration samples, e.g. greater than 5 vol. \% is not fabricated in this research.

The reduction of mechanical strength of samples with nickel flakes may stem from the morphology of the particles. Unlike the graphene which have a high strength and modulus in plane (Young's modulus of 1.0 TPa, intrinsic strength of $130 \mathrm{GPa}$ [32]), the nickel flakes have cracks and gaps while reaching a certain scale, as shown in Fig. 16b. Especially when these defects are not rapped by the cement hydration products, they result in stress concentration and then strength reduction.
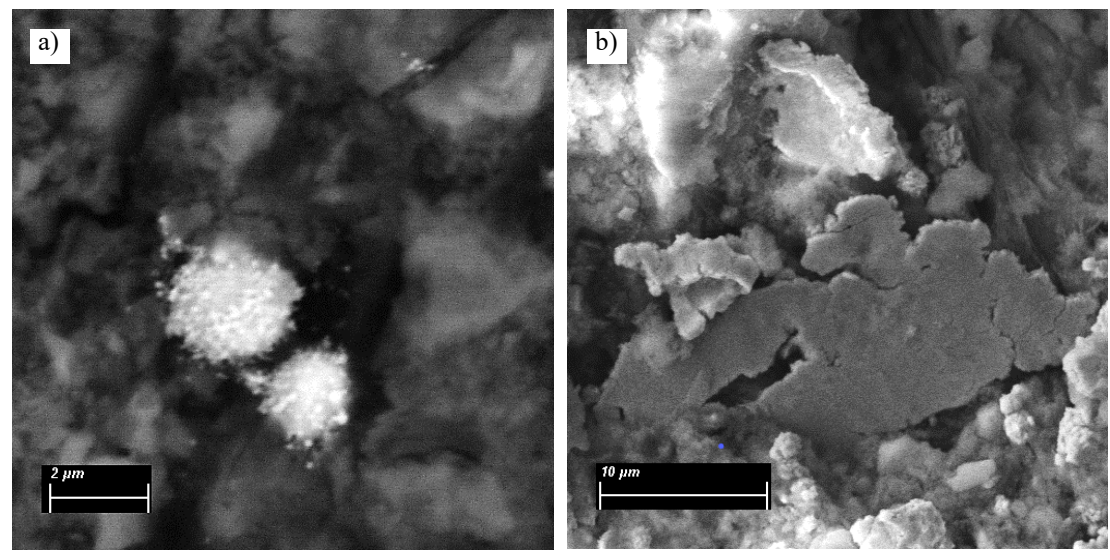

Fig. 16. a). Particle agglomerations of N50 nickel powder. b). The cracks and gaps on type F nickel flakes.

\subsubsection{Modulus of elasticity}


As shown in Fig. 17, the modulus of elasticity (MOE) of the samples has a similar trend with the compressive strength. The MOE of plain cement paste is $15.5 \mathrm{GPa}$. Composites with nickel flakes possess the lowest MOE due to the relatively low strength and high compressive deformation. It is followed by the samples with N50 nano-scale nickel powder, which is affected by the particle aggregations. It is worth noting that the MOE of samples with 5 vol.\% N500 nickel powder is 16.7 GPa, 7.7\% higher than the reference samples. This may be contribute to the well dispersed particulates and a high nickel elastic modulus (207 GPa [33]). Other samples present moderate MOE values, but in high content, e.g., 15 vol.\%, the MOE drops down. This could be caused by the high dose of surfactant when dispersing a great number of particles into water. The negative air entraining effect from the surfactant inevitably reduces the strength and MOE of the samples.

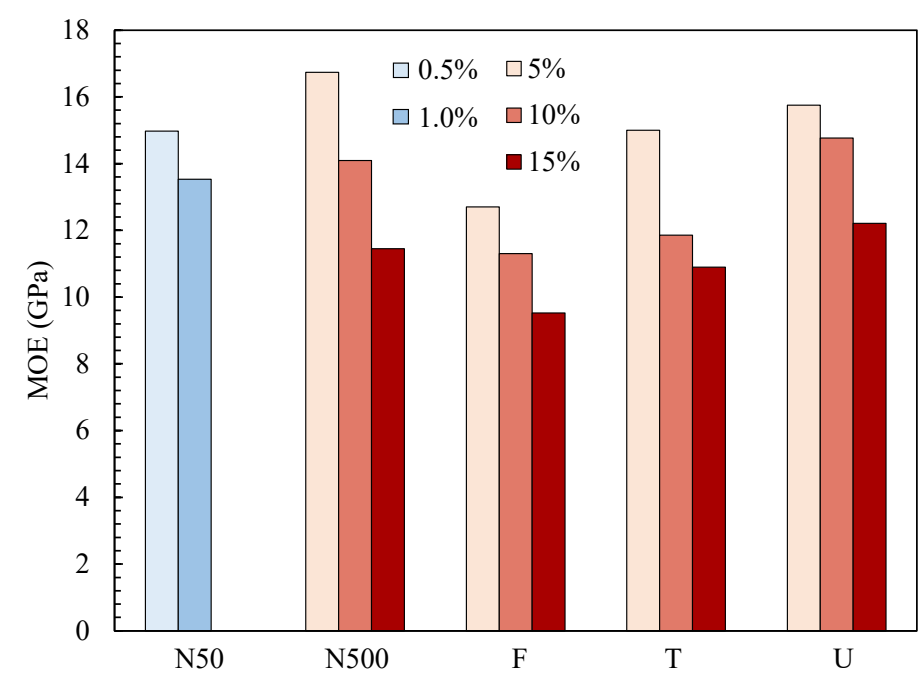

Fig. 17. The modulus of elasticity (MOE) of cement-based composites with different nickel powder in $0.1 \%, 0.5 \%, 5 \%, 10 \%$ and $15 \%$ volumes content. (The type $\mathrm{F}$ contents are $5 \%, 7.5 \%$ and $10 \%$ ).

\subsection{Sensitivity of the sensors (Gauge Factor)}

Gauge factor (GF) presents the sensitivity of the sensors, which is the FCR value per unit strain. High performance sensors can achieve great FCR values under small deformation when subjected to external 
loading. In this research, the FCR values are measured in the elastic range of the samples under cyclic compressive loading. Pre-loading is to guarantee the stability of contact resistance between the electrodes and matrix, and to eliminate tiny brittle in the first loading cycle. Two strain gauges are attached to the opposite sides of the cubic samples for balance purpose.

As shown in the statistical data in Fig. 18, half of the total 28 samples' GFs exceed 500. When the GF value is larger than 500, it is defined as giant piezoresistivity, indicating that the sensors show a significant piezoresistive performance [34]. In these samples with giant GF, two-thirds are aligned by magnetic field. The highest GF value is 3143 from 5 vol.\% T type nickel powder concentration with chain structure along $0.252 \mathrm{~T}$ magnetic field orientation, followed by samples with 10 vol.\% U type nickel and 5 vol.\% nickel flake, respectively, both of which exceed 1000. Most of the composites in this study have high FCR values within a small range of strain, especially after the conductive filler are aligned into chain structure. For practical application, the sensors with 5 vol.\% $\mathrm{T}$ type nickel powder has the greatest GF. The relative low filler concentration is benefit for particle dispersion and cost effectiveness. The sensor with 10 vol.\% U type has second large GF, but the content is higher than the T type. Although the unit price of latter is cheaper, the balance between these two kinds of samples still need further investigation. Regarding the sensors with 5 vol.\% nickel flakes, despite its relative low compressive strength, it still has the possibility to be used in the situations where no high stress is required. 


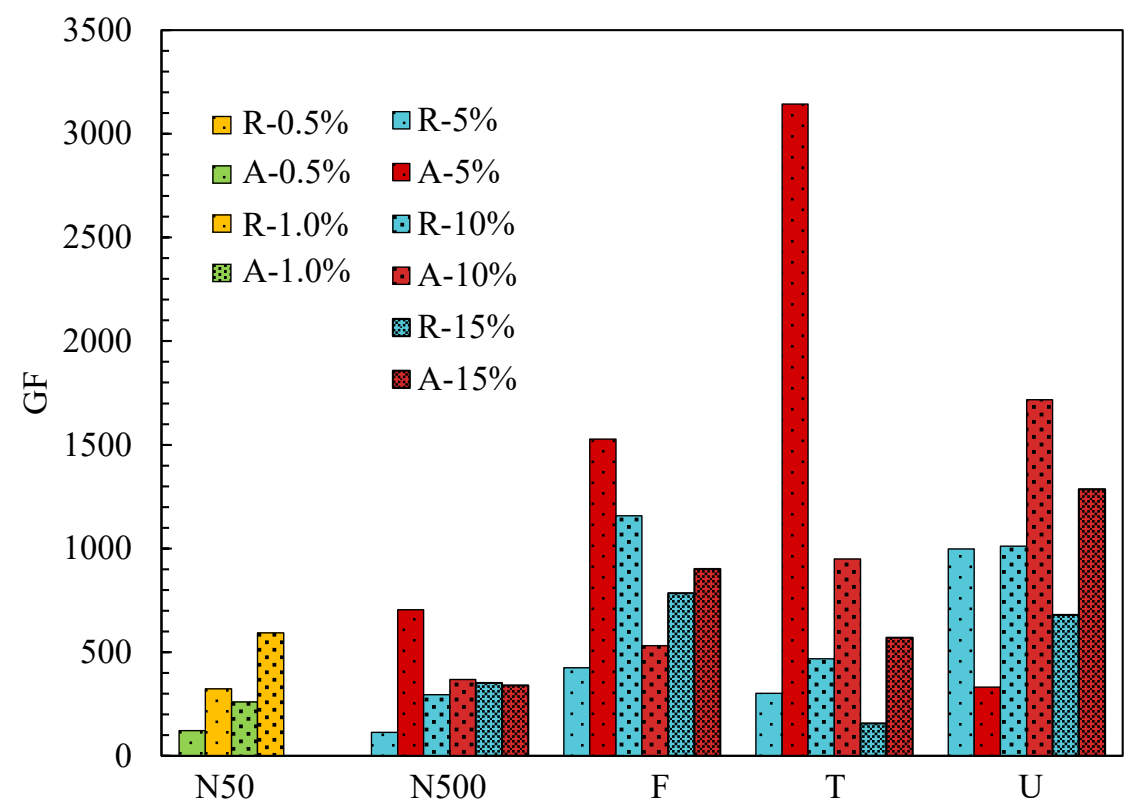

Fig. 18. Comparison of gauge factors in this research. (R-sample with random dispersed fillers, Asample with chain pattern particles in the direction of magnetic field, Type F nickel flakes concentration range from 5 vol. $\%, 7.5$ vol. $\%$ to 10 vol. $\%$ ).

Overall, the cement composites with different types of nickel powder aligned by magnetic field can be summarised below.

Table 3. The characteristics of cement sensors with magneto-aligned nickel powder in this research.

\begin{tabular}{cccl}
\hline $\begin{array}{c}\text { Nickel } \\
\text { powder }\end{array}$ & $\begin{array}{c}\text { Optimum } \\
\text { concentration }\end{array}$ & $\begin{array}{c}\text { Gauge } \\
\text { factor }\end{array}$ & \multicolumn{1}{c}{ Characteristic } \\
\hline N50 & $<1.0$ vol.\% & 593 & $\begin{array}{l}\text { Adequate piezoresistive performance at ultra-low } \\
\text { content; challenges in dispersing particles. }\end{array}$ \\
N500 & $<5$ vol.\% & 705 & $\begin{array}{l}\text { Low concentration filler, high mechanical strength } \\
\text { and MOE }\end{array}$ \\
F & 7.5 vol.\% & 531 & $\begin{array}{l}\text { Good conductivity and piezoresistivity, poor } \\
\text { mechanical properties, limited use (in elastic stress } \\
\text { region only) }\end{array}$ \\
T & 5 vol.\% & 3143 & $\begin{array}{l}\text { High sensitivity with relatively low filler } \\
\text { concentration }\end{array}$
\end{tabular}



U $\quad 10$ vol.\%
1717
Moderate sensitivity, high demand in particle
content

\section{Conclusions}

This study investigates the piezoresistive and mechanical properties of the cement-based composites with five types of nickel powders both in random distribution and aligned by magnetic field. The micro structure is examined by SEM. Each type of samples exhibits unique self-sensing performance. The sensitivity of the cement sensors with oriented nickel powder is improved in general. Conclusively, with the magneto-aligned particles, the cemebt-based sensors possess much higher piezoresitivity comparted to sensors with randomly dispersed particles. For anisotropic cement sensors, the particle morphology does have distinct impact on the piezoresistivity of the cement-based sensors. The general pattern is that fine particles can trigger useable piezoresistivity at ultra-low (N50) or low (N500) concentrations. While, small diameter of the particles brings challenges in particle dispersion during mixing process. The desirable particle shape is the spherical particles and samples with such shape particles have higher piezorestivity than non-spherical particles, such as flake shape. It is also worth mention that the spikey spherical particle can channel the conductive network more efficient than spherical shape ones.

The detailed conclusions can be summarised as follows:

(1) Both the anisotropic and isotropic piezoresistive performance increase with the decreasing particle size. The enhancement of magnetic fied on piezoresistivity reaches 2 to 3 times in the samples with particles in nano and submicron scale at lower concentration. The composites with $5 \mu \mathrm{m}$ nickel powder shows superior anisotropic piezoresistivity. Small particles with lower content has similar piezoresistive performance to the samples with large particles and higher concentration. 
(2) The piezoresistivity of composites with 5 vol.\% type T nickel particles is improved 6.2 times after the fillers are aligned to chain structure by magnetic field, having the highest piezoressitivity promotion factor in this study, which provides a great potential for fabricating cement sensors with high piezoresistive performance and cost effectiveness.

(3) The performance of N500 nickel powder and cement composite is second only to the Type samples. It is relatively easy to disperse into water, and according to the FCR curve charts, the filler content has the feasibility to be further decreased (under 5 vol.\%) under magnetic field.

(4) The sensors made by nickel flakes and U type nickel powder present good piezoresistive performance as well. The maxium FCRs are $-32 \%$ to $-20 \%$ under $10 \mathrm{MPa}$ cyclic compressive loading, respectively. However, the anisotropic piezoresistivity from the unidirectional conductive network shows no advantages compares to the samples with random distributed fillers.

(5) Nano-scale nickel particles give the cement sensors with a combined characteristic: ultra-low nickel filler concentration, e.g., below 0.1 vol. $\%$ and moderate FCR values. It has great application potential if the particle is fully dispersed.

(6) Regarding the sensitivity of the samples, half of the sensors' GF in this study exceed 500, most of which are magnetic field induced. The highest GF is 3143 from the samples with 5 vol.\% magnetoaligned type $\mathrm{T}$ nickel powder, followed by 5 vol.\% type $\mathrm{F}$ and 10 vol.\% type U magneto-aligned nickel powder, both of which exceed 1500 .

(7) The compressive strength of all the samples with lower concentration, e.g., 5 vol\%, is in a reasonable range for engineering application. With the increment of particle content to $15 \mathrm{vol} \%$, N500, F, $\mathrm{T}$ and $\mathrm{U}$ type nickel and cement composites lose workability and are with considerable low mechanical strength. Regarding the MOE, 5 vol.\% N500 and type U nickel powder cement sensors are slightly higher than the plain paste. Other samples with same concentration are lightly lower, but drop significantly after 10 vol.\%. 


\section{References}

1. Han, B., S. Ding, and X. Yu, Intrinsic self-sensing concrete and structures: A review. Measurement, 2015. 59: p. 110-128.

2. Han, B., et al., Nickel particle-based self-sensing pavement for vehicle detection. Measurement, 2011. 44(9): p. 1645-1650.

3. Reza, F., J.A. Yamamuro, and G.B. Batson, Electrical resistance change in compact tension specimens of carbon fiber cement composites. Cement and Concrete Composites, 2004. 26(7): p. 873-881.

4. Tian, Z., et al., A state-of-the-art on self-sensing concrete: Materials, fabrication and properties. Composites Part B, 2019. 177: p. 107437

5. Gao, J., et al., Dispersion of carbon fibers in cement-based composites with different mixing methods. Construction and Building Materials, 2017. 134: p. 220-227.

6. Azhari, F. and N. Banthia, Carbon Fiber-Reinforced Cementitious Composites for Tensile Strain Sensing. ACI Materials Journal, 2017. 114(1): p. 129-136.

7. Wen, S. and D.D.L. Chung, Piezoresistivity-Based Strain Sensing in Carbon Fiber-Reinforced Cement. ACI Materials Journal, 2007. 104(2): p. 171-179.

8. Yoo, D.-Y., et al., Effects of carbon nanomaterial type and amount on self-sensing capacity of cement paste. Measurement, 2019. 134: p. 750-761.

9. Azhari, F. and N. Banthia, Cement-based sensors with carbon fibers and carbon nanotubes for piezoresistive sensing. Cement and Concrete Composites, 2012. 34(7): p. 866-873.

10. Galao, O., et al., Strain and damage sensing properties on multifunctional cement composites with CNF admixture. Cement and Concrete Composites, 2014. 46: p. 90-98.

11. Wang, H., X. Gao, and R. Wang, The influence of rheological parameters of cement paste on the dispersion of carbon nanofibers and self-sensing performance. Construction and Building Materials, 2017. 134: p. 673-683.

12. Dong, W., et al., Structural response monitoring of concrete beam under flexural loading using smart carbon black/cement-based sensors. Smart Materials and Structures, 2020. 29(6): p. 065001.

13. Chen, L., et al., Synergistic effect of conductive carbon black and silica particles for improving the pyroresistive properties of high density polyethylene composites. Composites Part B: Engineering, 2019. 178: p. 107465.

14. Sun, S., et al., Nano graphite platelets-enabled piezoresistive cementitious composites for structural health monitoring. Construction and Building Materials, 2017. 136: p. 314-328.

15. Chen, M., et al., Mechanical and smart properties of carbon fiber and graphite conductive concrete for internal damage monitoring of structure. Construction and Building Materials, 2017. 142: p. 320327.

16. Belli, A., et al., Evaluating the Self-Sensing Ability of Cement Mortars Manufactured with Graphene Nanoplatelets, Virgin or Recycled Carbon Fibers through Piezoresistivity Tests. Sustainability, 2018. 10(11): p. 4013.

17. E. Ozbulut, O., Z. Jiang, and D. K. Harris, Exploring scalable fabrication of self-sensing cementitious composites with graphene nanoplatelets. Smart Materials and Structures, 2018. 27(11): p. 115029.

18. Dong, W., et al., Electrical resistivity and mechanical properties of cementitious composite incorporating conductive rubber fibres. Smart materials and structures, 2019. 28(8): p. 85013.

19. Ding, Y., et al., Effect of steel fiber and carbon black on the self-sensing ability of concrete cracks under bending. Construction and Building Materials, 2019. 207: p. 630-639.

20. Lee, S.Y., H.V. Le, and D.J. Kim, Self-stress sensing smart concrete containing fine steel slag aggregates and steel fibers under high compressive stress. Construction and Building Materials, 2019. 220: p. 149-160.

21. Han, B., B. Han, and X. Yu, Experimental study on the contribution of the quantum tunneling effect to the improvement of the conductivity and piezoresistivity of a nickel powder-filled cement-based composite. Smart Materials and Structures, 2009. 18(6): p. 065007. 
22. Han, B.G., B.Z. Han, and X. Yu, Effects of the content level and particle size of nickel powder on the piezoresistivity of cement-based composites/sensors. Smart Materials and Structures, 2010. 19(6): $p$. 065012.

23. Li, H., H.-g. Xiao, and J.-p. Ou, A study on mechanical and pressure-sensitive properties of cement mortar with nanophase materials. Cement and Concrete Research, 2004. 34(3): p. 435-438.

24. Yoo, D.Y., et al., Electrical and piezoresistive properties of cement composites with carbon nanomaterials. Journal of Composite Materials, 2018. 52(24): p. 3325-3340.

25. Al-Dahawi, A., et al., Effect of mixing methods on the electrical properties of cementitious composites incorporating different carbon-based materials. Construction and Building Materials, 2016. 104: $\mathrm{p}$. 160-168.

26. Kashif Ur Rehman, S., et al., Influence of Graphene Nanosheets on Rheology, Microstructure, Strength Development and Self-Sensing Properties of Cement Based Composites. Sustainability (2071-1050), 2018. 10(3): p. 822.

27. Li, Y., K. Wang, and Z. Su, Dispersed Sensing Networks in Nano-Engineered Polymer Composites: From Static Strain Measurement to Ultrasonic Wave Acquisition. Sensors (14248220), 2018. 18(5): p. 1398.

28. Chuah, S., et al., Nano reinforced cement and concrete composites and new perspective from graphene oxide. Construction and Building Materials, 2014. 73: p. 113-124.

29. Chen, L., Experimental study of ultra-sharp silicon nano-tips. Solid State Communications, 2007. 143(11): p. 553-557.

30. Han, B., X. Yu, and J. Ou, Chapter 9 - Nickel-Powder-Based Self-Sensing Concrete, in Self-Sensing Concrete in Smart Structures, B. Han, X. Yu, and J. Ou, Editors. 2014, Butterworth-Heinemann. p. 271-313.

31. Kirkpatrick, S., The nature of percolation 'channels'. Solid state communications, 1973. 12(12): p. 1279-1283.

32. Lee, C., et al., Measurement of the Elastic Properties and Intrinsic Strength of Monolayer Graphene. Science, 2008. 321(5887): p. 385-388.

33. Sharma, A., et al., Nickel nanoparticles set a new record of strength. Nature communications, 2018. 9(1): p. 4102-4102.

34. Chung, D.D.L., A critical review of piezoresistivity and its application in electrical-resistance-based strain sensing. Journal of Materials Science, 2020. 55(32): p. 15367-15396. 